Teil II -

Aufwachsen in überwachten Umgebungen:

Privatheit in Kita, Schule und Familie 



\title{
Das ist Privatsache! Zwischen Schutzbedarf und \\ Freiheitswunsch: Aufwachsen im digitalen Umfeld
}

\author{
Jutta Croll und Elena Frense
}

\begin{abstract}
Kinder wachsen heute in einer von (digitalen) Medien geprägten Lebenswelt auf. Die von Erwachsenen vorgenommene Differenzierung von analoger und digitaler Welt ist für sie keine relevante Trennlinie mehr. Insbesondere die Gewährleistung von Datenschutz und Privatsphäre wird im Spannungsfeld von Schutz- und Freiheitsrechten vor Herausforderungen gestellt. Unter Bezugnahme auf die UN-Kinderrechtskonvention analysiert der Beitrag die Regelungen der europäischen Datenschutz-Grundverordnung und deren Umsetzung im Hinblick auf den Vorrang des Kindeswohls gem. Art. 3 UN-KRK; er zeigt wie das Kinderrecht auf Beteiligung an Maßnahmen des Jugend- und Datenschutzes in der Praxis umgesetzt werden kann und welche Perspektive Kinder selbst auf Privatsphäre im digitalen Umfeld haben. Überlegungen, wie die Politik den Rahmen für eine ausgewogene Balance von Schutz und Freiheit und eine an der Lebenswelt Heranwachsender orientierte Erziehung setzen sollte, bilden den Abschluss der Ausführungen.
\end{abstract}

\section{Einleitung: Aufwachsen im digitalen Umfeld}

Kinder wachsen heute in einer von (digitalen) Medien geprägten Lebenswelt auf. Internet (97\%) und Smartphone (96\%) nutzen, Musik hören (93\%) und Online-Videos anschauen (84\%) führen laut JIM-Studie 2019 die Hitliste der regelmäßigen Medienbeschäftigungen Heranwachsender in ihrer Freizeit an (Medienpädagogischer Forschungsverbund Südwest 2020: 12-13). Freizeitaktivitäten wie Treffen mit Freund*innen und Bekannten oder Sport machen werden von drei Viertel bis zwei Drittel aller Jugendlichen mindestens mehrmals die Woche ausgeübt (Medienpädagogischer Forschungsverbund Südwest 2020: 10-11).

Die von Erwachsenen, d. h. von Eltern, pädagogischen Fachkräften, aber auch von Wissenschaftler*innen vorgenommene Differenzierung von 
analoger und digitaler Welt ist für Kinder ${ }^{1}$ heute keine relevante Trennlinie mehr. Kommunikation ist für sie gleich über welchen Kanal in allen Lebenssituationen unverzichtbar, Emotion ist nicht an körperliche Begegnung gebunden und soziale Nähe entfaltet sich (auch) im digitalen Raum. Dies stellt Erziehungsverantwortliche, aber auch Politik und Gesellschaft vor neue Herausforderungen.

Im Folgenden soll das Spannungsfeld von Schutz- und Freiheitsrechten mit einem Fokus auf Datenschutz und Privatsphäre näher beleuchtet werden. Den Rahmen setzen dabei die UN-Kinderrechtskonvention (UNKRK) und die Umsetzung der darin verbrieften Rechte einerseits und die Entwicklung und Etablierung von Nutzungsformen digital gestützter medialer Kommunikation und Interaktion sowie Produktion und Rezeption medialer Inhalte durch Kinder und Jugendliche andererseits. Dabei kann eine mit der Verabschiedung der UN-KRK und der Entstehung des WorldWideWeb im Jahr 1989 seit dreißig Jahren parallel verlaufende Entwicklung in den beiden Bereichen beobachtet werden, bei der bisher kaum erforscht ist, inwieweit mögliche Korrelationen und Wechselwirkungen bestehen.

Das Hans-Bredow-Institut hat 2006/2007 das deutsche Jugendschutzsystem, bestehend aus Jugendschutzgesetz (JuSchG) und Jugendmedienschutzstaatsvertrag (JMStV) der Länder evaluiert und in seinem Bericht formuliert: „Insgesamt wird man in diesem Bereich absolute Sicherheit weder versprechen noch erwarten können; es handelt sich um Risikomanagement.“ Diesen Begriff hat 2015 das Zentrum für Kinderschutz im Internet (I-KiZ) für die Ausrichtung seiner Arbeit aufgegriffen und sich mit der Frage befasst, wie den Risiken und Gefährdungen, denen Kinder und Jugendliche im Internet ausgesetzt sein können, durch eine intelligente, auf unterschiedliche Elemente gestützte Strategie, begegnet werden kann. Darauf soll im Folgenden näher eingegangen und die Anwendbarkeit eines solchen Konzepts im Bereich des Datenschutzes analysiert werden.

1 Der Begriff Kinder wird in diesem Artikel im Sinne der UN-KRK zur Bezeichnung von jungen Menschen, die das 18. Lebensjahr noch nicht vollendet haben, verwendet. 
2. Europäische Einordnung von DSGVO und UN-Kinderrechtskonvention: Bezüge, Widersprüche, Erwartungen und Potenziale

Das Konzept der Privatsphäre ist auch in der UN-Kinderrechtskonvention verankert. Art. 16 zum Schutz der Privatsphäre und Ehre lautet wie folgt:

(1) Kein Kind darf willkürlichen oder rechtswidrigen Eingriffen in sein Privatleben, seine Familie, seine Wohnung oder seinen Schriftverkehr oder rechtswidrigen Beeinträchtigungen seiner Ehre und seines Rufes ausgesetzt werden.

(2) Das Kind hat Anspruch auf rechtlichen Schutz gegen solche Eingriffe oder Beeinträchtigungen.

Diesem Ansatz ist die Ambivalenz von Freiheits- und Schutzrecht inhärent. Der Anspruch auf Privatsphäre - im öffentlichen Raum ebenso wie im familiären Umfeld - ist ein Freiheitsrecht, das angesichts der Risiken und Bedrohungen eben jener Privatsphäre - insbesondere in digitalen Räumen - einen besonderen Schutz erfordert.

\subsection{Kinderrechtlich relevante Regelungen der DSGVO}

Mit der Europäischen Datenschutz-Grundverordnung wird erstmals im Bereich des Datenschutzes ein altersdifferenzierender Ansatz verfolgt. Dabei stützt sich die DSGVO auf die UN-Kinderrechtskonvention und versteht Kinder als Personen, die das 18. Lebensjahr noch nicht vollendet haben. Sowohl in den Erwägungsgründen als auch im Normtext werden Kinder als eine Gruppe von Betroffenen mehrfach erwähnt, eine im Rahmen des Projektes Kinderschutz und Kinderrechte in der digitalen Welt durchgeführte Synopse ergab 35 unterschiedliche Fundstellen. Zudem werden besondere Anforderungen an die Verarbeitung der Daten von Kindern gestellt.

Die einleitende Formulierung des Erwägungsgrundes 38 „Kinder verdienen bei ihren personenbezogenen Daten besonderen Schutz" ist unter Organisationen und Individuen, die sich für das Kindeswohl einsetzen, auf eine breite positive Resonanz gestoßen, hat aber zugleich auch Fragen aufgeworfen, nach der Ausgewogenheit zwischen dem Schutzbedürfnis einerseits und dem Selbstbestimmungsrecht der betroffenen Kinder und Jugendlichen andererseits. Auch Erwägungsgrund 58 hebt die besondere Schutzwürdigkeit von Kindern hervor und fordert, dass „Informationen und Hinweise in einer dergestalt klaren und einfachen Sprache erfolgen, dass ein Kind sie verstehen kann", wenn sich die Datenverarbeitung an 
Kinder richtet. Daraus folgt, dass für die Datenverarbeitung in Kontexten, die sich nicht explizit an Kinder richten, aber oft von diesen genutzt werden, die Verständlichkeit für Kinder nicht ausdrücklich gefordert wird. Kindern wird in Art. 13 der UN-KRK das Recht auf freien Zugang zu Informationen verbrieft:

(1) Das Kind hat das Recht auf freie Meinungsäußerung; dieses Recht schließt die Freiheit ein, ungeachtet der Staatsgrenzen Informationen und Gedankengut jeder Art in Wort, Schrift oder Druck, durch Kunstwerke oder andere vom Kind gewählte Mittel sich zu beschaffen, zu empfangen und weiterzugeben.

Dieses Recht kann durch die in Erwägungsgrund 58 formulierte Beschränkung der Verständlichkeitsanforderung auf Kontexte, die sich direkt an Kinder richten, berührt sein.

In Erwägungsgrund 65 erfolgt unter dem Stichwort „Recht auf Vergessenwerden“ eine Abwägung zwischen dem Recht des Kindes auf Löschung von Daten, zu deren Verarbeitung es die Einwilligung im Kindesalter gegeben hat, und dem Recht anderer Personen auf freie Meinungsäußerung und Information. Letzterem Recht wird dabei der Vorrang gegeben und die Speicherung der personenbezogenen Daten, zu der das minderjährige Kind seine Zustimmung gegeben hatte, wird im Interesse der Informationsfreiheit Anderer für zulässig erklärt.

Erwägungsgrund 71 nimmt Bezug auf Entscheidungen und Maßnahmen, die ausschließlich auf automatisierter Verarbeitung personenbezogener Daten Betroffener beruhen, d. h. auf der Grundlage des sogenannten Profilings, und formuliert, dass derartige Maßnahmen „kein Kind betreffen [sollten]".

Erwägungsgrund 75 adressiert Risiken „für die Rechte und Freiheiten natürlicher Personen - mit unterschiedlicher Eintrittswahrscheinlichkeit und Schwere - die aus einer Verarbeitung personenbezogener Daten hervorgehen können, die zu einem physischen, materiellen oder immateriellen Schaden führen könnte, insbesondere [...] wenn personenbezogene Daten schutzbedürftiger natürlicher Personen, insbesondere Daten von Kindern“, verarbeitet werden. Mit Bezug auf die in den Erwägungsgründen 71 und 75 angeführten Maßnahmen wäre im Sinne von Art. 32 UNKRK (Schutz vor wirtschaftlicher Ausbeutung) eine Konkretisierung hinsichtlich des Profiling zu kommerziellen Zwecken zur Wahrung des Vorrangs des Kindeswohls wünschenswert.

Auch in den Formulierungen der Erwägungsgründe zeigt sich die Ambivalenz des Rechts auf Datenschutz und Privatsphäre, die schon in Art. 16 der UN-KRK festgestellt werden konnte. Dieses gleichermaßen als Frei- 
heits- und Schutzrecht angelegte Privileg kann zu konfligierenden Regelungen führen. Im Folgenden soll anhand einiger Bestimmungen der DSGVO, welche Kinder ausdrücklich erwähnen, näher auf Beispiele eingegangen werden.

Das Grundprinzip der DSGVO beruht darauf, Datenverarbeitung für unzulässig zu erklären und nur unter Ausnahmevorbehalt zuzulassen. In der Folge ist zunächst Art. $6^{2}$ vorrangig von Bedeutung. Art. 6 (1) b) erlaubt die Datenverarbeitung „für die Erfüllung eines Vertrags, dessen Vertragspartei die betroffene Person ist“. BGB Art. 108 (1) besagt: „Schließt der Minderjährige einen Vertrag ohne die erforderliche Einwilligung des gesetzlichen Vertreters, so hängt die Wirksamkeit des Vertrags von der Genehmigung des Vertreters ab. "Demzufolge wäre der durch eine minderjährige, d. h. unter 18 Jahre alte, Person mit einem Diensteanbieter durch die Registrierung auf dessen Plattform geschlossene Vertrag schwebend unwirksam, so lange diese Genehmigung nicht vorliegt. Daraus ergibt sich die Frage, welche Auswirkungen auf den Vorrang des Kindeswohls zu erwarten sind, wenn sich Diensteanbieter bei der Datenverarbeitung auf Art. 6 (1) b) stützen. Zugleich muss unter dem Primat des Kindeswohls untersucht werden, welche Unterschiede aus der Einwilligung gemäß Art. 6 (1) a) und in der Folge gemäß Art. 8 bei Personen unter 16 Jahren durch den Träger der elterlichen Verantwortung für das Kind einerseits und der Genehmigung des Vertrags gemäß Art. 6 (1) b) durch den gesetzli-

2 Art. 6: Rechtmäßigkeit der Verarbeitung (1) Die Verarbeitung ist nur rechtmäßig, wenn mindestens eine der nachstehenden Bedingungen erfüllt ist:

a) Die betroffene Person hat ihre Einwilligung zu der Verarbeitung der sie betreffenden personenbezogenen Daten für einen oder mehrere bestimmte Zwecke gegeben;

b) die Verarbeitung ist für die Erfüllung eines Vertrags, dessen Vertragspartei die betroffene Person ist, oder zur Durchführung vorvertraglicher Maßnahmen erforderlich, die auf Anfrage der betroffenen Person erfolgen;

c) die Verarbeitung ist zur Erfüllung einer rechtlichen Verpflichtung erforderlich, der der Verantwortliche unterliegt;

d) die Verarbeitung ist erforderlich, um lebenswichtige Interessen der betroffenen Person oder einer anderen natürlichen Person zu schützen;

e) die Verarbeitung ist für die Wahrnehmung einer Aufgabe erforderlich, die im öffentlichen Interesse liegt oder in Ausübung öffentlicher Gewalt erfolgt, die dem Verantwortlichen übertragen wurde;

f) die Verarbeitung ist zur Wahrung der berechtigten Interessen des Verantwortlichen oder eines Dritten erforderlich, sofern nicht die Interessen oder Grundrechte und Grundfreiheiten der betroffenen Person, die den Schutz personenbezogener Daten erfordern, überwiegen, insbesondere dann, wenn es sich bei der betroffenen Person um ein Kind handelt. 
chen Vertreter der minderjährigen Person (unter 18 Jahren) andererseits in Bezug auf das Kindeswohl resultieren können.

Art. 6 (1) f) gibt den Interessen, Grundrechten und Grundfreiheiten der betroffenen Person, die den Schutz personenbezogener Daten erfordern, wenn es sich um ein Kind handelt, den Vorrang vor der Wahrung der berechtigten Interessen des Verantwortlichen oder eines Dritten. Daraus resultiert die Frage, unter welchen Voraussetzungen der Vorrang des Kindeswohls als Interesse, Grundrecht oder Grundfreiheit im Sinne von Art. 6 (1) f) interpretiert werden darf.

In besonderem Maße manifestiert sich ein Konflikt von Schutz- und Freiheitsinteressen des Kindes in den Regelungen des Art. $8^{3}$ der DSGVO. Art. 8 legt als Altersgrenze für die erforderliche Einwilligung der Eltern in die Nutzung des Angebotes eines Dienstes der Informationsgesellschaft, das einem Kind direkt gemacht wird, die Vollendung des 16. Lebensjahres fest. Ab 16 Jahren können Kinder somit selbständig einwilligen, gelten aber bis 18 Jahre als Kind im Sinne der DSGVO. Die Datenschutz-Grundverordnung lässt in diesem Punkt den Mitgliedstaaten der EU einen Gestaltungsspielraum, die Altersgrenze in einem Korridor zwischen 13 und 16 Jahren festzulegen, um den Datenschutz an nationale Gegebenheiten anzupassen (sogenannte Öffnungsklausel). Hier ist unter Bezugnahme auf die Freiheitsrechte des Kindes in erster Linie zu klären, ob die Altersgrenze unter Berücksichtigung des Art. 5 der UN-KRK (Respektierung des Elternrechts) angemessen gesetzt ist. Im europäischen Vergleich sind die verschiedenen Länder hier zu unterschiedlichen Einschätzungen gekom-

3 Art 8: Bedingungen für die Einwilligung eines Kindes in Bezug auf Dienste der Informationsgesellschaft

(1) Gilt Artikel 6 Absatz 1 Buchstabe a bei einem Angebot von Diensten der Informationsgesellschaft, das einem Kind direkt gemacht wird, so ist die Verarbeitung der personenbezogenen Daten des Kindes rechtmäßig, wenn das Kind das sechzehnte Lebensjahr vollendet hat. Hat das Kind noch nicht das sechzehnte Lebensjahr vollendet, so ist diese Verarbeitung nur rechtmäßig, sofern und soweit diese Einwilligung durch den Träger der elterlichen Verantwortung für das Kind oder mit dessen Zustimmung erteilt wird. Die Mitgliedstaaten können durch Rechtsvorschriften zu diesen Zwecken eine niedrigere Altersgrenze vorsehen, die jedoch nicht unter dem vollendeten dreizehnten Lebensjahr liegen darf.

(2) Der Verantwortliche unternimmt unter Berücksichtigung der verfügbaren Technik angemessene Anstrengungen, um sich in solchen Fällen zu vergewissern, dass die Einwilligung durch den Träger der elterlichen Verantwortung für das Kind oder mit dessen Zustimmung erteilt wurde.

(3) Absatz 1 lässt das allgemeine Vertragsrecht der Mitgliedstaaten, wie etwa die Vorschriften zur Gültigkeit, zum Zustandekommen oder zu den Rechtsfolgen eines Vertrags in Bezug auf ein Kind, unberührt. 
men und es ist ein „Flickenteppich" von geltenden Altersgrenzen entstanden: In Deutschland gilt die Altersgrenze von 16 Jahren, in einigen europäischen Ländern hat man sich auf 13 Jahre geeinigt, in anderen gilt eine Altersgrenze von 14 oder 15 Jahren (Milkaite/Lievens 2019). Inwieweit bei der Entscheidung neben bestehenden Altersgrenzen in anderen Rechtsbereichen auch kinderrechtliche Erwägungen einbezogen wurden, kann nicht abschließend beantwortet werden. Ebenso bleibt offen, inwieweit Art. 2 der UN-KRK (Achtung der Kindesrechte; Diskriminierungsverbot) durch diese unterschiedlichen Regeln berührt ist. Dies gilt umso mehr, da bislang Uneinigkeit darüber besteht, ob für die Anwendung der Altersgrenze das Herkunftslandprinzip gilt, d. h. es wäre die Altersgrenze des Landes, in dem das Unternehmen angesiedelt ist, geltend, oder ob hier das Marktortprinzip greift und damit jeweils die Altersgrenze des Landes, in dem das Unternehmen seine Leistungen am Markt erbringt, was für Kinder in Europa zu Ungleichbehandlung führt.

Unter Berücksichtigung von Art. 5 der UN-KRK zur Respektierung des Elternrechts ${ }^{4}$ stellt sich die Frage, in welchen Fällen die Einwilligungserfordernis nach Art. 8 DSGVO im Widerspruch zum Vorrang des Kindeswohls steht. Hier ist beispielsweise an die Nutzung von Online-Beratungsdiensten von Kindern in familiären Konfliktsituationen zu denken und an den gemäß SGB VIII Art. 8 (3) $)^{5}$ Kindern zustehenden Anspruch auf Beratung ohne Kenntnis der Personensorgeberechtigten. Im Sinne der Lebensweltorientierung sozialer Arbeit muss dieser Anspruch heute auch für die Nutzung von Diensten der Informationsgesellschaft für Beratungszwecke gelten.

Grundsätzlich kommt aber die Einwilligungserfordernis nach Art. 8 nur zum Tragen, wenn die datenverarbeitende Stelle die Rechtmäßigkeit der Verarbeitung auf Art. 6, (1) a) „Einwilligung“ beruft. Bei Datenverarbeitung gestützt auf Art. 6 (1) b) - f) ist die elterliche Einwilligung nicht erforderlich. Welche positiven oder negativen Auswirkungen die Entscheidung eines Diensteanbieters, sich auf Art. 6 (1) b) -f) zu berufen, auf den Vorrang des Kindeswohls hat, bedarf ebenso der näheren Betrachtung wie die

4 Art. 5: Die Vertragsstaaten achten die Aufgaben, Rechte und Pflichten der Eltern oder gegebenenfalls, soweit nach Ortsbrauch vorgesehen, der Mitglieder der weiteren Familie oder der Gemeinschaft, des Vormunds oder anderer für das Kind gesetzlich verantwortlicher Personen, das Kind bei der Ausübung der in diesem Übereinkommen anerkannten Rechte in einer seiner Entwicklung entsprechenden Weise angemessen zu leiten und zu führen.

5 SGB VIII Art. 8 (3): Kinder und Jugendliche haben Anspruch auf Beratung ohne Kenntnis des Personensorgeberechtigten. 
Frage, welche Auswirkungen auf den Vorrang des Kindeswohls sich daraus ergeben können, dass der Diensteanbieter als die datenverarbeitende Stelle die Daten differenziert und sich auf unterschiedliche Abschnitte von Art. 6 (1) beruft, z. B. Verarbeitung allgemeiner Nutzerdaten gestützt auf Art 6 (1) b) oder f), sensible Daten nach Art. 9 gestützt auf Art. 6 (1) a).

Aus den vorstehenden Ausführungen wird deutlich, dass eine abschließende Beurteilung, inwieweit die Regelungen der DSGVO in Einklang zu bringen sind mit dem Vorrang des Kindeswohls gemäß Art. 3 der UNKRK weiterer Untersuchungen bedarf. Die ausdrücklich auf Kinder Bezug nehmenden Regelungen der DSGVO stellen - wie zuvor dargelegt - die Schutzbedürftigkeit von Kindern in den Vordergrund und bewirken so eine Nachrangigkeit des legitimen Freiheitsanspruchs von Kindern.

\subsection{Konvention 108 und die Leitlinien des Europarats zur Achtung, zum Schutz und zur Verwirklichung der Rechte des Kindes im digitalen Umfeld}

Der Europarat hat mit dem „Übereinkommen zum Schutz des Menschen bei der automatischen Verarbeitung personenbezogener Daten“, der so genannten Konvention 108 bereits 1985 den ersten - und noch immer einzigen - internationalen Vertrag über das Recht des Einzelnen auf den Schutz seiner persönlichen Daten zur Ratifizierung freigegeben und damit frühzeitig auf die zunehmende Bedeutung automatisierter Prozesse für den Schutz personenbezogener Daten hingewiesen. Wenngleich die Regelungen der Konvention 108 deutlich weniger detailliert sind als die der Datenschutzgrundverordnung, ist die Bedeutung des Vertrags durch die Unterzeichnung der Mitgliedstaaten des Europarats und den Beitritt von weiteren neun Nicht-Mitgliedstaaten durchaus weitreichend und auf internationaler Ebene von Relevanz. Mit dem am 18. Mai 2018 vom Ministerkomitee des Europarats verabschiedeten Änderungsprotokoll Konvention 108+ wird angestrebt, den Herausforderungen, welche die Verwendung neuer Informations- und Kommunikationstechnologien für den Schutz der Privatsphäre darstellen, zu begegnen sowie die Umsetzung der Konvention wirksam zu stärken. Zu den vorgenommenen Modernisierungen zählen u. a. die Definition möglicher Rechtsgrundlagen der Datenverarbeitung und die Einführung des Prinzips der Datenminimierung sowie die Berücksichtigung von genetischen und biometrischen Daten als sensible Daten gemäß Art. 6. Darüber hinaus wurde Art. 7 im Hinblick auf die Datensicherheit um Meldepflichten im Fall von Verstößen ergänzt, und die Betroffenenrechte auf Auskunft, Änderung und Löschung wurden gestärkt. Datenverarbeitenden Stellen wird eine Rechenschaftspflicht auferlegt und sie 
werden zur Implementierung des Prinzips Safety by Design verpflichtet. Schließlich wird dem grenzüberschreitenden Datenverkehr mehr Beachtung geschenkt, und es werden Regelungen eingeführt, um den Schutz personenbezogener Daten auch in diesem Fall zu gewährleisten; die Rolle der Datenschutzaufsichtsbehörden ist um Informations- und Aufklärungsaufgaben erweitert.

Art. 1 der Konvention 108 hebt bereits in der ersten Fassung die Abhängigkeit der Wahrnehmung der Grundrechte und Freiheiten des Menschen von der Gewährleistung des Schutzes seiner Privatsphäre hervor. Dieser menschenrechtliche Ansatz prägt auch die Leitlinien des Europarats zur Achtung, zum Schutz und zur Verwirklichung der Rechte des Kindes im digitalen Umfeld, die parallel zum Änderungsprotokoll der Konvention 108+ von einer Arbeitsgruppe des Europarats entwickelt und am 4. Juli 2018 vom Ministerkomitee verabschiedet wurden. Ausgangspunkt dieser Entwicklung ist die Sofia-Strategie des Europarats, mit der im Jahr 2016 erstmals eine Strategie zur Umsetzung der UN-KRK vom Europarat beschlossen wurde, die neben den Säulen Chancengleichheit, Teilhabe und Partizipation, gewaltfreies Leben und kindgerechte Justiz auch das digitale Umfeld berücksichtigt. Dem Ad hoc Committee for the Rights of the ChildCAHENF wurde die Arbeitsgruppe CAHENF-IT zur Seite gestellt, mit dem Auftrag, entsprechende Leitlinien für das digitale Umfeld zu erarbeiten. Die Leitlinien befassen sich im Abschnitt 3.4 zu Datenschutz und Privatsphäre in den Art. 26 bis 39 mit kinderrechtlichen Aspekten dieses Grundrechts. Sie adressieren den Schutz der Privatsphäre des Kindes im öffentlichen wie im privaten Bereich, stellen Anforderungen an die kindgerechte Information und Aufklärung sowie die Einbeziehung von Kindern, formulieren einen Auftrag zur Berücksichtigung des Kindeswohls bei Technikgestaltung und Voreinstellungen sowie einen Anspruch des Kindes auf anonyme und pseudonyme Nutzungsmöglichkeiten. Mit der in Art. 37 formulierten Forderung, „das Profiling von Kindern, d.h. jede Form der automatisierten Verarbeitung personenbezogener Daten, die darin besteht, ein ,Profil' eines Kindes anzulegen, insbesondere um Entscheidungen über das Kind zu treffen oder seine persönlichen Vorlieben, Verhaltensweisen und Einstellungen zu analysieren oder vorherzusagen," gesetzlich zu verbieten, gehen die Leitlinien des Europarats über die gesetzlichen Anforderungen der DSGVO hinaus. 
Für die Abwägung der Balance von Schutz- und Freiheitsrechten ist die in Art. $31^{6}$ formulierte Anforderung, bei jeglicher Datenverarbeitung eine Bewertung der möglichen Auswirkungen und der Risiken in Bezug auf die Beeinträchtigung der Rechte des Kindes vorzunehmen, besonders relevant; denn damit wird der zuvor bereits dargelegten Ambivalenz des Schutzund Freiheitsrechts auf Privatsphäre in den Leitlinien ausdrücklich Rechnung getragen (Europarat 2018/2019: 16-18).

Im Folgenden soll näher darauf eingegangen werden, wie der auf politischer Ebene formulierte Anspruch durch die Beteiligung von Kindern und Jugendlichen verwirklicht und so die Perspektive der Betroffenen selbst in politisches Handeln einbezogen werden kann.

\section{Beteiligung von Kindern an sie betreffenden Angelegenheiten}

Wie zuvor ausgeführt ist die Beteiligung von Kindern in den einschlägigen Normen zum Datenschutz praktisch nicht vorgesehen. Lediglich die Leitlinien des Europarats bieten - gestützt auf die UN-KRK - einen Anknüpfungspunkt. Im folgenden Abschnitt werden Grundlagen und Möglichkeiten der Beteiligung von Kindern an sie betreffenden Angelegenheiten und Maßnahmen am Beispiel des Jugendmedienschutzes aufgezeigt.

\subsection{Warum: Grundlagen in der UN-KRK}

In der UN-KRK von 1989 sind die Beteiligungsrechte von Kindern fest verankert. Art. 12 und 13 sehen vor, dass Kinder in allen sie betreffenden Angelegenheiten anzuhören sind und ihre Meinung angemessen und entsprechend ihrem Alter und ihrer Reife zu berücksichtigen ist. Allerdings werden die Kindern zustehenden Beteiligungsrechte in der Gesetzgebungspraxis bislang nur selten berücksichtigt. Erstmals soll im Rahmen der aktuellen Novellierung des Jugendschutzgesetzes im Jahr 2020 auch gesetzlich verankert werden, dass Kinder an der Entwicklung sie betreffender Schutzmaßnahmen zu beteiligen sind. Dabei stützt sich der Entwurf des JuSchG auf die UN-KRK und sieht Jugendschutz als Resultat von Maßnahmen in

6 Art. 31: Die Staaten sollten sicherstellen, dass potenzielle Auswirkungen der beabsichtigten Datenverarbeitung auf die Rechte des Kindes bewertet werden und dass die Datenverarbeitung so konzipiert ist, dass das Risiko einer Beeinträchtigung dieser Rechte vermieden oder minimiert wird. 
einem Dreieck aus Schutz, Befähigung und Teilhabe, bei dem das Kindeswohl im Mittelpunkt steht. Auf die Wechselwirkung zwischen den Elementen des Dreiecks wird abschließend noch näher eingegangen.

\subsection{Wie: Methoden der Kinderbeteiligung}

Dass und wie Kinder an der Erarbeitung von Fragen des Jugendmedienschutzes beteiligt werden können, zeigt eine aktuelle Studie von Frense (2020). In ihrer empirischen Untersuchung hat sie mit Sechst- und Zehntklässler*innen Handlungsempfehlungen für einen zeitgemäßen Jugendmedienschutz erarbeitet. Das zugrundeliegende Paradigma Kinderschutz durch Partizipation und Befähigung, angelehnt u.a. an Liebel (2009: 33), Lansdown (2005: 39ff.), Feinstein und O'Kane (2009: 5), illustriert, dass es gelingen kann, Kinder an der Entwicklung effektiver Schutzstrategien zu beteiligen. Zentral ist es dabei, Räume zu schaffen, in denen Kinder als Expert*innen ihrer Lebenswelt ernst genommen werden, ihnen Gehör verschafft und ihnen auf Augenhöhe begegnet wird. Dabei sind stets die sich entwickelnden Fähigkeiten (evolving capacities) der Kinder zu berücksichtigen. Dies kann beispielsweise im Rahmen von Workshops geschehen, die inhaltlich nah an der medialen Lebenswelt der Heranwachsenden orientiert sind. Basis für diese Art der Beteiligung stellt die Befähigung dar, um die Kinder in die Lage zu versetzen, informierte Entscheidungen zu treffen.

„Aber was braucht es, um Kinder zu befähigen? [...] Information ist die Voraussetzung für tatsächliche Beteiligung, denn nur wer informiert ist, kann substanziell mitreden.“ (Radlicki 2011: 18)

Neben der Information ist auch die Befähigung zur Reflexion des eigenen (Medien-)Handelns und der daraus resultierenden Konsequenzen und Implikationen zentral. Dafür bieten sich Ansätze an, die klassischerweise in den Bereich der Medienpädagogik fallen. Was es braucht sind also seitens der Diensteanbieter Bezüge und Schnittstellen zur Medienpädagogik und seitens erwachsener Entscheidungsträger en Willen, Kinder aktiv in die Ausgestaltung von Schutzmaßnahmen einzubeziehen, was auch Ausdruck einer demokratischen Gesellschaft ist:

„Es ist an den Erwachsenen zu prüfen, ob sie die Anliegen von Kindern ernsthaft hören wollen und, ob ihnen tatsächlich Instrumente und Selbstvertrauen gegeben werden, sich für ihre Dinge zu engagieren. / Demokratie bemisst sich auch daran, wie gut sie die Stimme der 
Schwächsten hört. Für mich gehören starke und befähigte Kinder und ihre Stimme essenziell zu einer demokratischen Gesellschaft." (Radlicki 2011: 17)

Anhand empirischer Daten wird im nächsten Abschnitt die Perspektive von Kindern selbst auf Privatsphäre und Datenschutz als ein ihnen zustehendes Freiheits- und Schutzrecht dargelegt.

\section{Die Perspektive der Kinder}

Heranwachsende haben heute - in Abhängigkeit von ihren sich entwickelnden Fähigkeiten (evolving capacities) - schon früh ein Bewusstsein für den eigenen Anspruch auf Privatsphäre und die aus der Onlinenutzung und dem eigenen Handeln potenziell resultierenden Gefährdungen. Das Thema Privatheit wird für sie insbesondere dann bedeutend, wenn diese nicht respektiert wird. Laut DIVSI U25-Studie (2014 und 2018) vermeiden Kinder im Alter von neun bis 13 Jahren, um ihre Privatsphäre zu schützen vor allem die Angabe von Klarnamen und Adressen sowie die Veröffentlichung von Fotos, auf denen sie selbst abgebildet sind. Möglicher Datenmissbrauch im Internet oder die kommerzielle Verwertung persönlicher Informationen werden erst im späteren Altersverlauf als Risiken wahrgenommen. Die Altersgruppe der 14- bis 17-Jährigen definiert als privat vorrangig alles, was in den Bereich des Intimen und Peinlichen fällt, so zum Beispiel Informationen rund um Gefühle, Sorgen oder Ängste. Allgemeine personenbezogene Daten wie Geburtsdatum, Wohnort oder Schule hingegen werden von dieser Altersgruppe als weniger problematisch eingestuft und somit auch häufiger preisgegeben. Ein Verständnis für den Wert dieser Daten, insbesondere im Zusammenhang mit der Analyse des individuellen Nutzungsverhaltes zu kommerziellen Zwecken, ist kaum vorhanden. Als Instrument des Selbstschutzes werden entsprechende Privatsphäre-Einstellungen in Online-Communitys für ausreichend erachtet. $79 \%$ der 16bis 18-Jährigen, aber nur ein Viertel der 10- bis 11-Jährigen haben laut einer Studie des BITKOM (2014) individuelle Privatsphäre-Einstellungen in den von ihnen genutzten sozialen Netzwerken vorgenommen. Damit die Kinder neben dem Bewusstsein für Privatheit und die eigene Privatsphäre im Netz auch aktiv Maßnahmen zum Selbstschutz ergreifen, bedarf es offensichtlich sowohl eines gewissen Reifegrades und Alters als auch der umfassenden Vermittlung von Kenntnissen im Bereich des Datenschutzes und der Medienkompetenz (Croll/Pohle 2018). 
Im Rahmen des Projektes Kinderschutz und Kinderrechte in der digitalen Welt wurden 2018 Kinder mittels einer nicht repräsentativen Online-Befragung7 um ihre Meinung zu Aspekten des Datenschutzes gebeten. Die große Mehrheit der Kinder betont in dieser auf die Beziehung und Abgrenzung zu ihren Eltern fokussierenden Erhebung den Anspruch auf Schutz der Privatsphäre im familiären Umfeld hinsichtlich ihrer Online-Kommunikationsaktivitäten. In Bezug auf das eigene Handeln stellt sie der verantwortungsbewusste Umgang mit persönlichen Daten vor das Dilemma, unter Umständen nur einen eingeschränkten oder gar keinen Zugang zu für sie wichtigen Online-Angeboten und Diensten zu haben. In diesem Fall tendieren sie entweder zu einer pragmatischen Entscheidung für die Preisgabe der geforderten Daten und nehmen den damit einhergehenden Verlust an Privatsphäre in Kauf oder entwickeln Lösungsstrategien wie die Verwendung von Fake-Namen oder eigens für solche Zwecke angelegten sogenannten „Spam-E-Mail-Adressen“.

Ein zentrales Anliegen der von Frense (2020) befragten jungen Menschen ist die auch in Erwägungsgrund 58 der DSGVO geforderte kindgerechte Verständlichkeit von Datenschutzinformationen, wie die folgenden Zitate zeigen:

„Sowas wie AGBs oder so. Wenn da jetzt irgendwie stehen würde, Ihre geographische Lage wird jetzt in selektiertem Blablabla irgendwas'. Sowas wird da bestimmt gemacht, weil die es ja rechtlich korrekt machen müssen, aber für den User ist es besser, wenn dann so gesagt wird: 'Ihr Standort wird jetzt gezeigt. Sie können ibn an- und ausmachen und für diese an und für diese nicht." (männlich, 16 Jahre)

„[A]uch so jugendgerechte Kommunikation zwischen Hersteller und Nutzer [...] also, dass Jugendliche jetzt nicht in den Appstore gehen müssen und dann wirklich so die ganzen AGBs durchlesen müssen oder irgendwelche Informationen durchlesen müssen. Dass man das dann wirklich auf der App erscheint, dass das für die Kinder einfacher ist." (männlich, 16 Jahre)

Hintergrund ist dabei oft die persönliche Erfahrung mit mangelhaften Privatsphäre-Einstellungen, da Datenschutzbedingungen und Allgemeine Ge-

7 Insgesamt haben sich im Zeitraum vom 17. April bis 7. Mai 2018221 Kinder unter https://kinderrechte.digital sowie www.kindersache.de beteiligt. Weitere 24 Schülerinnen und Schüler einer sechsten Klasse aus Berlin haben im Rahmen einer Unterrichtsstunde teilgenommen und den Kurz-Fragebogen, der geschlossene Fragen und jeweils drei Antwortmöglichkeiten zur Auswahl anbot, ausgefüllt sowie teilweise ergänzend eigene Kommentare zu den Fragen abgegeben. 
schäftsbedingungen (AGB) vielfach als nutzer*innenunfreundlich wahrgenommen werden und beklagt wird, dass seitens der Diensteanbieter Updates nicht transparent kommuniziert werden.

"[S]chon am Anfang, als es die Funktion neu gab, da habe ich gar nicht gecheckt, dass ich meinen Standort an hatte und dann [...] hatte ich den irgendwie am Anfang an und ich wollte den eigentlich gar nicht an haben. Und dann habe ich das irgendwie erst eine Woche später oder so mitbekommen, dass ich den überhaupt an hab. Keine Ahnung, das hätten die irgendwie am Anfang vielleicht deutlicher sagen können, fand ich jetzt so." (weiblich, 15 Jahre)

Daneben wurde in den Befragungen ein Spannungsverhältnis zwischen Forderungen nach Autonomie und individueller Verantwortung bezüglich des Umgangs mit Risiken deutlich. Die individuelle Verantwortung wurde sogar derart weit interpretiert, dass in Situationen von Privatsphäre- und/ oder anderen Rechtsverletzungen dem Individuum und nicht den Anbietern der Plattform die Verantwortung zugesprochen wird. (Frense 2020: 61)

„Letztendlich ist es ja die eigene Entscheidung, wenn man die Videos veröffentlicht oder seinen Account öffentlich stellt. Das ist ja bei jedem selbst. Also man kann ja auch bei TikTok einfach nur so TikToks machen, die dann privat sind und die man sich nur selbst angucken kann. Also von daher ist es dann ja sozusagen die eigene Schuld, wenn man es dann hochlädt auf seinem Account und irgendwie veröffentlicht." (weiblich, 16 Jahre)

Weitere Forderungen von Kindern und Jugendlichen wie ein Recht auf Löschen im Internet und die Kontrolle von dessen Einhaltung sowie eine Zustimmungspflicht bei der Veröffentlichung von Bildern der Kinder im Netz wurden im Rahmen des Netzfestes der re:publica 2019 mit Kindern erarbeitet (Croll 2019).

Diese mit unterschiedlichen Methoden erhobenen Perspektiven von Kindern auf ihre Rechte gemäß Art. 16 der UN-KRK zeigen ein breites Spektrum von Haltungen gegenüber Risiken und Verletzungen der Privatsphäre im digitalen Umfeld und sie zeugen von einem hohen Bewusstsein für die Notwendigkeit von Maßnahmen des Schutzes und der Eigenverantwortlichkeit. 


\section{Fazit und Ausblick: Das Recht auf Privatsphäre unter Druck im Digitalen}

Wenn Kinder im digitalen Umfeld aufwachsen, geschieht das in einem Spannungsfeld zwischen Schutzbedarf und Freiheitswunsch. Dies sollte eine an der Lebenswelt orientierte Erziehung (Thiersch 2014) berücksichtigen und die Politik dafür den geeigneten Rahmen setzen.

Die im Modell des Intelligenten Risikomanagements für verschiedene Altersgruppen definierten strategischen Schutzziele Risikoausschluss, Risikovermeidung und Risikoreduzierung spiegeln die Entwicklung entlang der Kindheits- und Jugendphasen wider. Je jünger die Kinder sind, umso mehr sollen Instrumente des Jugendmedienschutzes Risiken ausschließen oder vermeiden. Mit zunehmendem Alter der Kinder und Jugendlichen kann sich der Jugendmedienschutz auf die Reduzierung (gravierender) Risiken beschränken und den Fokus auf die Befähigung zum Umgang mit Risiken richten. Dieses Modell ist auf den Bereich des Datenschutzes übertragbar. Dazu bedarf es allerdings sowohl der Einhaltung der Vorgaben der DSGVO in Bezug auf Transparenz und Verständlichkeit, als auch der Förderung der Kompetenzen in Bezug auf den Datenschutz bei Kindern ebenso wie bei den für sie Verantwortung tragenden Erwachsenen.

Wird für Befähigungsmaßnahmen ein partizipativer Ansatz gewählt, d. h. die Kinder werden selbst an der Entscheidung über zu adressierende Risiken und der Entwicklung von geeigneten Schutzkonzepten beteiligt, darf erwartet werden, dass sie diese in höherem Maße akzeptieren. Die bisher eingesetzten Instrumente des Jugendschutzes und des Datenschutzes werden insbesondere von jungen Menschen im Teenageralter eher als Herausforderung gesehen, die es zu umgehen gilt. Gerade deshalb ist die Einbeziehung von Jugendlichen in die Entwicklung von Schutzmaßnahmen wichtig. Aus der Partizipation heraus wächst das Interesse und das Verständnis für die Notwendigkeit des Schutzes, Maßnahmen, an deren Entwicklung junge Menschen mitwirken, haben das Potenzial, in der Zielgruppe größere Akzeptanz zu finden. So kann mittels partizipativer Methoden ein effektiver Jugendmedienschutz gestaltet werden, der an der Lebenswelt und den Bedarfen von Heranwachsenden ansetzt.

Wie zuvor ausgeführt orientiert sich der aktuelle Prozess der Novellierung des JuSchG an der UN-KRK und sieht einen Jugendmedienschutz vor, der sich auf Art. 3 der UN-KRK stützt und das Kindeswohl in den Mittelpunkt eines Dreiecks aus Schutz, Befähigung und Teilhabe stellt (Croll 2018: 45). Schutz und Befähigung sind dabei die Grundlagen für gesellschaftliche Teilhabe, wobei sich Teilhabe wiederum positiv auf die Bereitschaft zur Akzeptanz von Schutz- und Befähigungsmaßnahmen auswirkt. In den Regelungen der DSGVO hingegen scheint teilweise eine diesen 
pro-partizipativen Entwicklungen zuwiderlaufende und paternalistisch vor allem auf Schutzmaßnahmen setzende Haltung auf. Diese steht insbesondere im Widerspruch zu dem in Art. 12 der UN-KRK verbrieften Recht des Kindes gehört zu werden. Ein potenziell aus der Berücksichtigung des Kindeswillens einerseits und dem Vorrang des Kindeswohls andererseits resultierendes Dilemma muss zukünftig auch im Bereich des Datenschutzes noch aufgelöst werden.

\section{Literatur}

BITKOM - Bundesverband Informationswirtschaft, Telekommunikation und neue Medien e. V. (Hg.) (2014): Jung und vernetzt-Kinder und Jugendliche in der digitalen Gesellschaft. Berlin. Online verfügbar unter: https://www.bitkom.org/sites/def ault/files/pdf/noindex/Publikationen/2014/Studien/Jung-und-vernetzt-Kinder-un d-Jugendliche-in-der-digitalen-Gesellschaft/BITKOM-Studie-Jung-und-vernetzt-2 014.pdf (Abfrage am: 25.05.2020).

Croll, Jutta (2019): Wo sind die Stimmen der Kinder in der Netzpolitik. Kinderschutz und Kinderrechte in der digitalen Welt. Online verfügbar unter: http://kinderrecht e.digital/fokus/index.cfm/key.3443 (Abfrage am: 13.04.2020).

Croll, Jutta (2019): Das Recht des Kindes auf Privatsphäre in einer digitalisierten Lebenswelt. In: Frühe Kindheit (2), S. 24-31.

Croll, Jutta (2018): Im Mittelpunkt das Kind. Eine kinderrechtliche Perspektive auf den Kinder- und Jugendschutz im Internet. In: Aus Politik und Zeitgeschichte 68 (40/41), S. 40-46.

Croll, Jutta / Pohle, Sophie (2018): Stopp! Geheim - Das Kinderrecht auf Datenschutz und Privatsphäre in der digitalen Welt. In: Merz Wissenschaft Kinder|Medien| Rechte 62 (6), S. 29-40.

Deutsches Institut für Vertrauen und Sicherheit im Internet (2018): DIVSI U25-Studie - Euphorie war gestern. Online verfügbar unter: https://www.divsi.de/wp-cont ent/uploads/2018/11/DIVSI-U25-Studie-euphorie.pdf (Abfrage am: 25.05.2020).

Deutsches Institut für Vertrauen und Sicherheit im Internet (DIVSI) (2014): DIVSIU25-Studie-Kinder, Jugendliche und junge Erwachsene in der digitalen Welt. Online verfügbar unter: https:/www.divsi.de/wp-content/uploads/2014/02/DIVSI-U25-S tudie.pdf (Abfrage am: 25.05.2020).

Europarat (2018/2019): Leitlinien zur Achtung, zum Schutz und zur Verwirklichung der Rechte des Kindes im digitalen Umfeld. Online verfügbar unter: https://rm.coe.i $\mathrm{nt} / 168092 \mathrm{dd} 25$ (Abfrage am: 03.06.2019).

Feinstein, Clare / O'Kane, Claire (2009): Children's and Adolescents' Participation and Protection from Sexual Abuse and Exploitation. No. 2009/09. New York: UNICEF Innocenti Research Centre. https://doi.org/10.18356/443a21a8-en. 
Frense, Elena (2020): Partizipativer Jugendmedienschutz: Anforderungen an einen zeitgemäßen Jugendmedienschutz aus Perspektive von Kindern und Jugendlichen. Frankfurt am Main: Debus Pädagogik.

Lansdown, Gerison (2005): The Evolving Capacities of the Child. Florence: Unicef Innocenti Research Centre.

Liebel, Manfred (2009): Kinderrechte - Aus Kindersicht: Wie Kinder weltweit zu ihrem Recht kommen. Berlin: Lit.

Medienpädagogischer Forschungsverbund Südwest (Hg.) (2020): JIM-Studie 2019Jugend, Information, Medien - Basisuntersuchung zum Medienumgang 12- bis 19-Jähriger. Online verfügbar unter: https:/www.mpfs.de/fileadmin/files/Studien/JIM/ 2019/JIM_2019.pdf (Abfrage am: 24.05.2020).

Milkaite, Ingrida / Lievens, Eva (2019): Better Internet for Kids-Status Quo regarding the Child's Article 8 GDPR Age of Consent for Data Processing across the EU. Better Internet for Kids. Online verfügbar unter: https://www.betterinternetforkids.eu/ $\mathrm{web} / \mathrm{portal} / \mathrm{practice} / \mathrm{aw}$ areness/detail? articleId=3017751 (Abfrage am: 25.05.2020).

Radlicki, Eva (2011): Wie ernst ist es uns mit dem „Kinder ernst nehmen"? In: Schächter, Markus / Apel, Peter (Hg.): Ich kann. Ich darf. Ich will: Chancen und Grenzen sinnvoller Kinderbeteiligung. Baden-Baden: Nomos, S. 17-20.

Thiersch, Hans (2014): Lebensweltorientierte Soziale Arbeit: Aufgaben der Praxis im sozialen Wandel (Edition Soziale Arbeit) (9. Auflage). Weinheim Basel: Beltz Juventa. 
